\title{
Zones of large and small values for Dedekind sums
}

\author{
by \\ KuRT GIRSTMAIR (Innsbruck)
}

1. Introduction and main results. Throughout this paper let $m$ and $N$ be integers, $N \neq 0$, with $(m, N)=1$. The classical Dedekind sum $s(m, N)$ is defined by

$$
s(m, N)=\sum_{k=1}^{|N|}((k / N))((m k / N))
$$

where $((\ldots))$ denotes the usual sawtooth-function (cf., e.g., [2]). Because of

$$
s(m,-N)=s(m, N) \text { and } s(m+N, N)=s(m, N),
$$

it suffices to consider $N \geq 1$ and $m$ in the range $0 \leq m<N$. The general definition, however, will be needed below. In the present context it is more natural to work with

$$
S(m, N)=12 s(m, N) .
$$

This paper deals with zones of large and small values of $|S(m, N)|$ for $m$ in the aforesaid range. To this end we observe, first,

$$
|S(m, N)|<N
$$

for all possible integers $m$ (cf., e.g., [5, (14)]). Our distinction between "large" and "small" is oriented towards the quadratic mean value of $S(m, N)$. It is known that

$$
\left(\frac{1}{N} \sum_{0 \leq m<N}|S(m, N)|^{2}\right)^{1 / 2} \asymp N^{1 / 2}
$$

for $N$ tending to infinity (more precisely, the asymptotic main term of (2) lies between $2 \sqrt{N}$ and $5 \sqrt{N}$, cf. [9]). Having (2) in mind we say that $S(m, N)$ is small if $S(m, N) \ll \sqrt{N}$ and large if $\sqrt{N}=o(S(m, N))$ as $N \rightarrow \infty$. It has been observed by various authors (cf. [2], [4], [5]) that $S(m, N)$ becomes large for arguments $m$ lying near points $N \cdot c / d$, where $d$ is a small natural number and $(c, d)=1$. In [5] we conjectured a sort of converse, namely, that $S(m, N)$ is small (in the above sense) if $m$ is outside a certain union

2000 Mathematics Subject Classification: Primary 11F20. 
of intervals with mid-points $N \cdot c / d, 1 \leq d \leq \sqrt{N}$. In this paper we prove a stronger version of this conjecture (cf. Theorem 1 ). Indeed, the intervals considered here are smaller than those of [5] (cf. the remark at the end of Section 2 below), and their definition is simpler.

The following terminology will be used: A Farey point (or simply an $F$-point) has the shape $N \cdot c / d, 1 \leq d \leq \sqrt{N}, 0 \leq c \leq d,(c, d)=1$. The denominator $d$ is called the order of the $F$-point. Further, we fix an arbitrary constant $C>0$. The interval

$$
I_{c / d}=\left\{x: 0 \leq x \leq N,|x-N \cdot c / d| \leq C \sqrt{N} / d^{2}\right\}
$$

is called the $F$-neighbourhood of the point $N \cdot c / d$. We write

$$
\mathcal{F}_{d}=\bigcup_{\substack{0 \leq c \leq d \\(c, d)=1}} I_{c / d}
$$

for the union of all neighbourhoods belonging to $F$-points of a fixed order $d$. Further,

$$
\mathcal{F}=\bigcup_{1 \leq d \leq \sqrt{N}} \mathcal{F}_{d}
$$

The integers $m$ (relatively prime to $N$, as always) lying in $\mathcal{F}$ are called $F$-neighbours. More precisely, $m$ is an $F$-neighbour of order $d$ if $m \in \mathcal{F}_{d}$, and it is an $F$-neighbour of $N \cdot c / d$ if it lies in $I_{c / d}$. An integer $m, 0 \leq m<N$, which is not in $\mathcal{F}$ is called an ordinary integer.

Theorem 1. Let $N \geq 15$ and $m$ be an ordinary integer. Then

$$
|S(m, N)| \leq(2+1 / C) \sqrt{N}+5 .
$$

It is not hard to see that the set $\mathcal{F}$ is small in terms of its Lebesgue measure: By (3) and (4), the measure of $\mathcal{F}_{d}$ is $\leq 2 C \varphi(d) \sqrt{N} / d^{2}$; accordingly, the measure of $\mathcal{F}$ is

$$
\leq 2 C \sqrt{N} \sum_{1 \leq d \leq \sqrt{N}} \varphi(d) / d^{2}=\frac{6 C}{\pi^{2}} \sqrt{N}(\log N+O(1))
$$

for large numbers $N$ (cf. [1, p. 71]). Nevertheless, the number of $F$-points might be large, since $\mathcal{F}$ is the union of many intervals - their number amounts to $\asymp N$. The following theorem says that this is not the case. In particular, the number of ordinary integers exceeds that of $F$-neighbours by far, which justifies our choice of names.

TheOREM 2. For each $N \geq 17$ the number of F-neighbours is

$$
\leq C \sqrt{N}(\log N+2 \log 2) .
$$

Let us look briefly at the graph

$$
G=\{(m, S(m, N)): 0 \leq m<N\}
$$


of the function $m \mapsto S(m, N)$. Theorems 1 and 2 say that $G$ consists of a very large flat zone outside of $\mathcal{F}$. This is illustrated by Diagram 1 , which represents all 1772 pairs $(m, S(m, N))$ with $m \notin \mathcal{F}$ for $N=2997=3^{4} \cdot 37$ and $C=1$. But the said flat zone is interrupted by many potential zones of disturbance, namely, the $F$-neighbourhoods. Since we used small circles to represent points $(m, S(m, N))$, only the largest of these neighbourhoods become visible in this diagram, the smaller ones disappear in the cluster of circles.

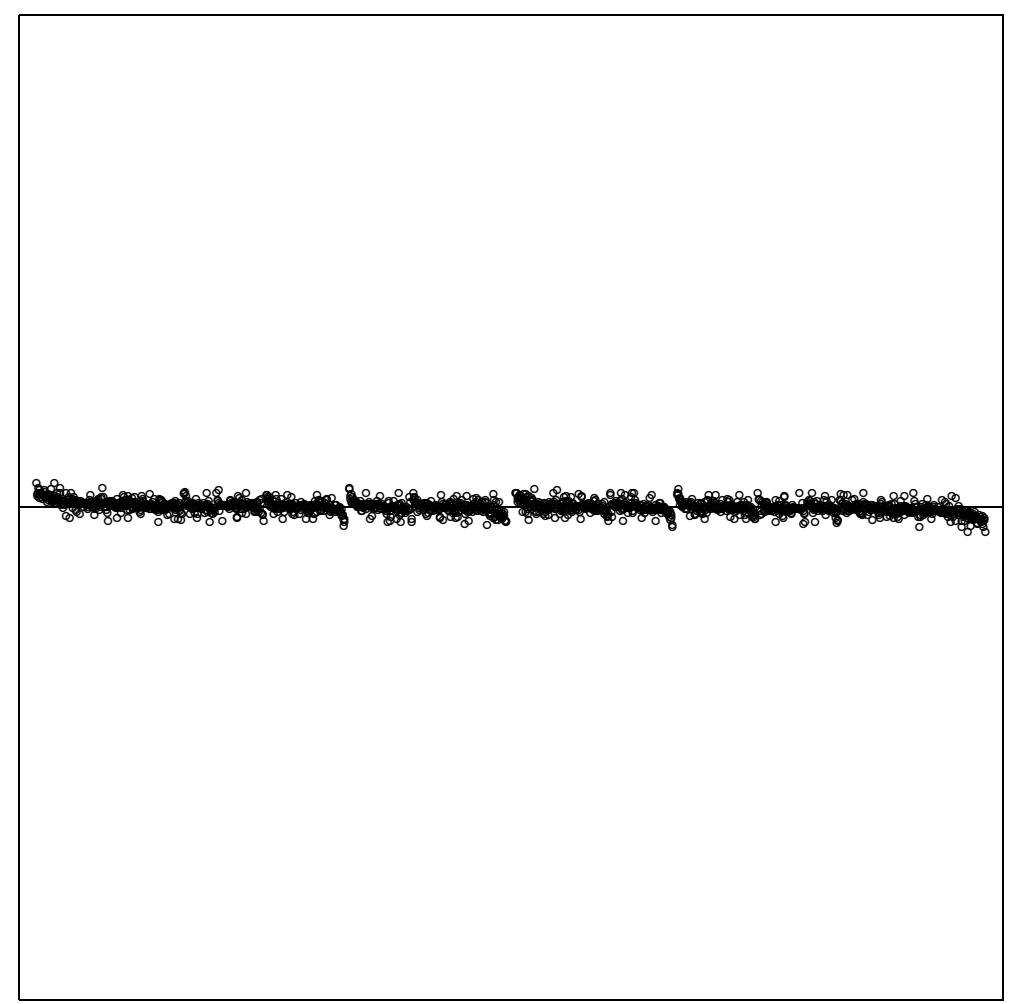

Diagram 1. The graph $G$ outside of $\mathcal{F}$ for $N=2997, C=1$

Are the $F$-neighbourhoods really zones of disturbance for the graph $G$ ? The answer is of an asymptotic nature, of course. Hence suppose that $N$ runs through a sequence of natural numbers tending to infinity. The number $d \leq \sqrt{N}$ need not be constant but may also tend to infinity, and the same is true of the number $m, 0 \leq m<N$. Suppose that $m$ remains an $F$-neighbour of order $d$ while $N$ grows. This means that the abscissa

$$
x_{m}=m-N \cdot c / d
$$

of $m$ relative to the corresponding $F$-point fulfils $x_{m} \ll \sqrt{N} / d^{2}$. We say $m$ is a distant $F$-neighbour (close $F$-neighbour, respectively) of order $d$ if 
$\left|x_{m}\right| \asymp \sqrt{N} / d^{2}\left(x_{m}=o\left(\sqrt{N} / d^{2}\right)\right.$, respectively $)$. In accordance with this notion we call an interval $I$ with mid-point $N \cdot c / d$ a close $F$-neighbourhood of order $d$ if its length is $o\left(\sqrt{N} / d^{2}\right)$ for $N$ tending to infinity.

THEOREM 3. (a) If, in the above setting, $m$ is a distant F-neighbour, then $S(m, N)$ is small, i.e., $S(m, N) \ll \sqrt{N}$.

(b) If, on the other hand, $m$ is a close F-neighbour of order $d$, then $S(m, N)$ is large. More precisely,

$$
S(m, N)=\frac{N}{d^{2} x_{m}}+o(\sqrt{N}) \quad \text { and } \quad \sqrt{N}=o\left(\frac{N}{d^{2} x_{m}}\right) .
$$

In view of Theorems 1 and 2, assertion (a) is not surprising. Indeed, if we change the constant $C$, an ordinary integer $m$ becomes an $F$-neighbour and conversely - so the asymptotic behaviour of distant $F$-neighbours and ordinary integers should be much the same. However, we combine this assertion with another observation: Since an $F$-neighbour $m$ of order $d$ is an integer, we have $\left|x_{m}\right| \geq 1 / d$, by (6). Suppose that $d \asymp \sqrt{N}$ as $N$ tends to infinity. Then $1 / d \asymp \sqrt{N} / d^{2}$, so $m$ remains distant in the above sense. Hence we obtain

Corollary 1. If $m$ is an F-neighbour of order $d \asymp \sqrt{N}$, then $S(m, N)$ $\ll \sqrt{N}$ for $N$ tending to infinity.

The corollary says that $F$-neighbourhoods of an order $d \asymp \sqrt{N}$ are not really zones of disturbance for the graph $G$. In fact, $|S(m, N)|$ may be considerably larger than $\sqrt{N}$ only if both $d=o(\sqrt{N})$ and $m$ is a close $F$-neighbour of order $d$. In view of (6), assertion (b) of Theorem 3 may be stated as follows:

Corollary 2. Let $N$ tend to infinity and $d=o(\sqrt{N})$. Let I run through a sequence of close $F$-neighbourhoods of order $d$. Then the points $(m, S(m, N)), m \in I$, of the above graph $G$ tend to the corresponding points $(x, y), x=m$, on the hyperbola

$$
(x-N \cdot c / d) \cdot y=N / d^{2} .
$$

The hyperbolic nature of the graph $G$ in the vicinity of $F$-points of small order has been observed in the literature (cf. [2], [4], [5]). The hyperbola of Corollary 2 is equilateral, its mid-point is the $F$-point $N \cdot c / d$, its asymptotes are given by $x=N \cdot c / d$ and $y=0$, and its parameter is $\sqrt{2 N} / d$. One should, however, not think that the part $\{(m, S(m, N)): m \in I\}$ of the graph has a symmetric shape relative to the mid-point of the hyperbola, since the distribution of right and left $F$-neighbours $m$ (i.e., those with $x_{m}>0$ and $x_{m}<0$, respectively) around $N \cdot c / d$ is in general not symmetric. In Section 3 we shall discuss some more details of this kind. 
Diagram 2 displays almost all pairs $(m, S(m, N))$ with $m \in \mathcal{F}$ for the same $N=2997$ and $C=1$. There are only two exceptions: the values $m=1$ and $m=N-1$ have been omitted for reasons of space, since $S(m, N)$ is close to $\pm N$ in these cases, whereas all other values do not exceed $\pm N / 2$.

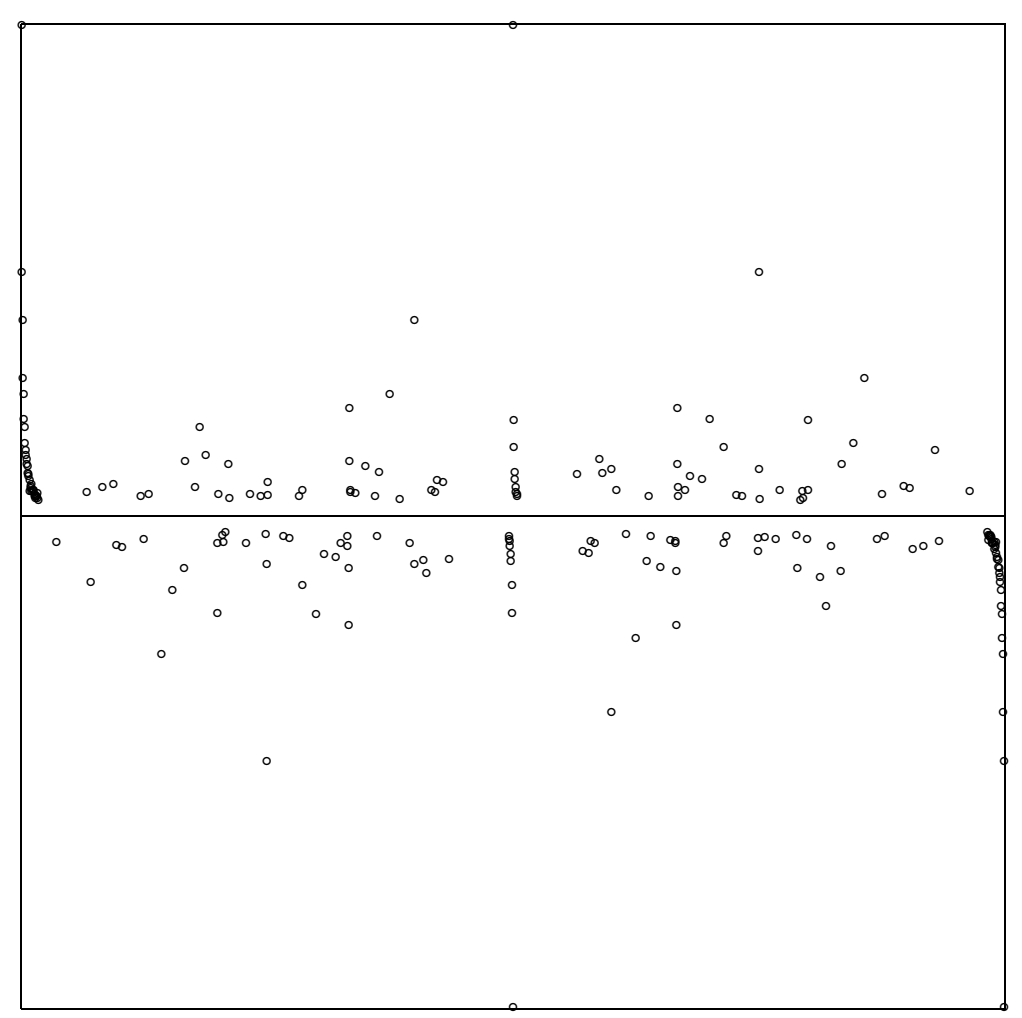

Diagram 2. The graph $G$ restricted to $\mathcal{F}$ for $N=2997, C=1$

Our understanding of "large" and "small" comes from a quadratic mean value (cf. (2)) which favours large numbers, of course. From this point of view the majority of Dedekind sums is not only small but even "microscopic". Indeed,

$$
\frac{1}{N} \sum_{0 \leq m<N}|S(m, N)| \ll \log ^{2} N
$$

(cf. [4, Lemma 6], cf. also [8]). The microscopic size of most Dedekind sums has some influence on $S(m, N)$ for distant $F$-neighbours $m$; namely, the hyperbolic shape of the above graph $G$ in general extends over those $m$, too - up to few exceptions, cf. the example and the remark at the end of Section 3. 
The distribution of Dedekind sums has attracted a great deal of interest (cf. [8], [2], [3], [4], [9]). The results of this paper belong to the easier ones. In our opinion they contribute something to the understanding of pictures of the graph $G$.

2. Farey neighbours and ordinary integers. In this section we prove Theorems 1 and 2. As above, let $N$ and $d$ be natural numbers with $d<N$, and $m$ and $c$ integers with $(m, N)=(c, d)=1$. We put

$$
q=m d-N c,
$$

which means $m-N \cdot c / d=q / d$. Then $q \neq 0$, for otherwise $m / N=c / d$, which is impossible since $d<N$ and both fractions are reduced. A crucial ingredient of all proofs is the generalized reciprocity law for Dedekind sums, which we state as follows (cf., e.g., [5, Lemma 1]): For some integer $r$ with $(r, q)=1$,

$$
S(m, N)=S(c, d) \pm S(r, q)+\frac{N^{2}+d^{2}+q^{2}}{N d q} \pm 3,
$$

where the $\pm \operatorname{sign}$ is the sign of $q$ in both cases. Combined with (1), the reciprocity law gives

$$
|S(m, N)| \leq d+|q|+\frac{N}{d|q|}+\frac{d}{N|q|}+\frac{|q|}{N d}+3
$$

for all $m, N, c, d$, and $q$ as above.

Proof of Theorem 1. Suppose now, in addition, that $c / d$ is an $F$-point and $0 \leq m<N$. Because of $d<N$, we have $N \geq 2$ and $m>0$. Further,

$$
d \leq \sqrt{N} \text { and } \frac{d}{N|q|} \leq \frac{d}{N} \leq \frac{1}{\sqrt{N}}
$$

By [6, p. 127, Theorem 10.5], there is always an $F$-point $c / d$ such that

$$
\left|\frac{m}{N}-\frac{c}{d}\right|<\frac{1}{d\lfloor\sqrt{N}\rfloor} .
$$

We fix such an $F$-point and obtain, from (8),

$$
|q| \leq \frac{N}{\sqrt{N}-1}=\sqrt{N}+1+\frac{1}{\sqrt{N}-1}
$$

and

$$
\frac{|q|}{d N} \leq \frac{|q|}{N} \leq \frac{1}{\sqrt{N}}+\frac{2}{N}
$$

provided that $N \geq 4$. Finally, suppose that $m$ is an ordinary integer. So its distance to the above $F$-point satisfies $|m-N \cdot c / d|>C \sqrt{N} / d^{2}$, which 
means $|q| \geq C \sqrt{N} / d$ and

$$
\frac{N}{d|q|} \leq \sqrt{N} / C
$$

On inserting the estimates (11)-(14) into (10), we obtain

$$
|S(m, N)| \leq\left(2+\frac{1}{C}\right) \sqrt{N}+4+\frac{1}{\sqrt{N}-1}+\frac{2}{\sqrt{N}}+\frac{2}{N},
$$

which is $\leq(2+1 / C) \sqrt{N}+5$ for $N \geq 15$.

The proof of Theorem 2 is based on the following

Lemma 1. Let $1 \leq d<N$. Then

$$
\sharp\left(\mathcal{F}_{d} \cap\{m \in \mathbb{Z}:(m, N)=1\}\right) \leq 2 C \sqrt{N} / d .
$$

Proof. Put $\delta=(N, d)$, so $N=\delta \cdot N^{\prime}, d=\delta \cdot d^{\prime}$ with $\left(N^{\prime}, d^{\prime}\right)=1$. Consider an element $m$ of the set in question. Since $m$ is in $\mathcal{F}_{d}$, there is an integer $q$ with $|q| \leq C \sqrt{N} / d$ such that $m d \equiv q \bmod N$ (cf. (8)). Then $\delta$ divides $q$, so $q=\delta \cdot q^{\prime}$ for some integer $q^{\prime}$ with $\left|q^{\prime}\right| \leq C \sqrt{N} /(\delta d)$. Further, $m d^{\prime} \equiv q^{\prime} \bmod N^{\prime}$ and $q^{\prime} \neq 0$ since, otherwise, $q=0$ and $N \mid d$, which is impossible. Let $m^{\prime}$ be the unique solution of the congruence $m d^{\prime} \equiv q^{\prime} \bmod N^{\prime}$ that lies in $\left\{0,1, \ldots, N^{\prime}-1\right\}$. Then $m$ has the shape

$$
m=m^{\prime}+l \cdot N^{\prime}
$$

for some $l \in \mathbb{Z}, 0 \leq l<\delta$. Since we have at most $2 C \sqrt{N} /(\delta d)$ possibilities for $q^{\prime}$, the assertion follows.

Proof of Theorem 2. The set of $F$-neighbours is

$$
\mathcal{F} \cap\{m \in \mathbb{Z}:(m, N)=1\} .
$$

By (5) and Lemma 1, its cardinality is bounded by

$$
\sum_{1 \leq d \leq \sqrt{N}} 2 C \sqrt{N} / d=2 C \sqrt{N} \sum_{1 \leq d \leq \sqrt{N}} 1 / d .
$$

However, one readily infers from [7, p. 6, Theorem 5] that the sum on the right hand side is $\leq \log (2 \sqrt{N})$ whenever $N \geq 17$.

REMARK. In [5] we considered intervals around the $F$-points which were larger than our $F$-neighbourhoods when the order $d \leq \sqrt{N}$ was large, their size being (roughly) $\sqrt{N / d^{3}}$. Altogether, those intervals contained $\asymp N^{2 / 3}$ integers, in contrast with the situation of Theorem 2 .

3. The behaviour of Farey neighbours. In what follows let $m$ be an $F$-neighbour of order $d$, so $d \leq \sqrt{N}$ and $q=m d-N c$ fulfils $|q| \leq C \sqrt{N} / d$ for the corresponding $F$-point $N \cdot c / d$. Then (9) gives

$$
S(m, N)=\frac{N}{d q}+E(d+|q|+4)
$$


if $N$ is sufficiently large, where $E(x)$ denotes an error term of absolute value $\leq x$.

Proof of Theorem 3. From (15) we clearly obtain

$$
S(m, N)=\frac{N}{d q}+E((1+C) \sqrt{N}+4)
$$

for large numbers $N$. If $m$ remains distant while $N$ tends to infinity, then $|q| \asymp \sqrt{N} / d$ and $N /(d|q|) \asymp \sqrt{N}$, so (16) shows $S(m, N) \ll \sqrt{N}$, which is assertion (a) of Theorem 3 .

As to assertion (b), suppose that $m$ remains a close $F$-neighbour. Then $x_{m}=o\left(\sqrt{N} / d^{2}\right)$ and $1=o\left(\sqrt{N} /\left(d^{2} x_{m}\right)\right)$. So the main term $N /(d q)=$ $N /\left(d^{2} x_{m}\right)$ of (15) satisfies $\sqrt{N}=o\left(N /\left(d^{2} x_{m}\right)\right)$. Further, $q=o(\sqrt{N} / d)$, so both $q=o(\sqrt{N})$ and $d=o(\sqrt{N})$. Altogether, the error term in (15) is $o(\sqrt{N})$.

As in Section 1, let us have a look at the graph $G$ of the function $m \mapsto S(m, N)$. We concentrate upon one particular order $d \geq 2$ with $d=o(\sqrt{N})$, which means that close $F$-neighbours $m$ of order $d$ are possible. The corresponding set $\mathcal{F}_{d}$ consists of $\varphi(d)$ (pairwise disjoint) intervals $I_{c / d}$, and Theorem $3(\mathrm{~b})$ says that close to the center $N \cdot c / d$ of $I_{c / d}$ the graph becomes similar to the hyperbola

$$
y=\frac{N}{d^{2}(x-N \cdot c / d)} .
$$

Accordingly, $G$ has a positive spike on the right and a negative one on the left of $N \cdot c / d$. The possible height (or depth) of these spikes is asymptotically bounded by $N /(d \delta)$ with $\delta=(N, d)$. Indeed, since $|q|=|m d-N c| \geq \delta$, the asymptotic value of $|S(m, N)|$ is $|N /(d q)| \leq N /(d \delta)$. If, for instance, $\delta=1$, then the whole set $\mathcal{F}_{d}$ contains exactly one $m$ with $q=1$, so the (asymptotically) maximal height $N / d$ is taken for exactly one integer $m \in \mathcal{F}_{d}$; the same holds for the depth $-N / d$. The case $d=1$ is exceptional inasmuch as we have two intervals $I_{0}$ and $I_{1}$ instead, each of which defines only one branch of the respective hyperbola.

However, the similarity of $G$ with the said hyperbolas is restricted by the fact that the distribution of numbers $m,(m, N)=1$, in the set $\mathcal{F}_{d}$ may not be uniform. If the order $d$ grows, the $F$-neighbourhoods $I_{c / d}$ contain fewer of these integers and become empty with increasing frequency: In fact, $\mathcal{F}_{d}$ contains at most $2 C \sqrt{N} / d$ such integers, by Lemma 1 . So each $F$-neighbourhood $I_{c / d}$ contains

$$
\leq \frac{2 C \sqrt{N}}{d \varphi(d)}
$$

numbers $m$ on average. This mean value tends to zero if $d \gg N^{1 / 4+\varepsilon}$, say. 
Moreover, the integers $m$ are not symmetric about the center $N \cdot c / d$ of $I_{c / d}$ in general, as the following trivial example shows: If $I_{c / d}$ contains both a right $F$-neighbour $m$ and a left $F$-neighbour $m^{\prime}$, then

$$
1 \leq m-m^{\prime} \leq 2 C \sqrt{N} / d^{2},
$$

which requires $d \ll N^{1 / 4}$. For the same reason, $m^{\prime}$ is relatively far away from the center $N \cdot c / d$ if $m$ is close to it. Thus, if $x_{m}=1 / d$, say, then $\left|x_{m^{\prime}}\right| \geq 1-1 / d$.

EXAmPle. Let $N=2 \cdot 10^{6}+3$ (a prime number), $d=11, c=7$, so the corresponding $F$-point is $\approx 1272729.182$. Choosing $C=1$, we have $C \sqrt{N} / d^{2} \approx 11.688$. Hence $I_{c / d}$ contains 23 integers $m$ with $(m, N)=1$ (viz., all of $1272718, \ldots, 1272740)$. For these numbers $m,|q| \leq\lfloor\sqrt{N} / 11\rfloor=$ 128 , so (15) shows that $S(m, N)=N /(d q)+E(143)$; and going back to (9) one even obtains $E(142.001)$. Because of $N /(d|q|) \geq 1420.45, S(m, N)$ must be equal to $N /(d q)$ up to a relative error of less than 10 percent. Thus, the main term of Theorem $3(\mathrm{~b})$ is essentially the correct value of $S(m, N)$ for all $22 F$-neighbours of $N \cdot 7 / 11$. On computing the exact values of $S(m, N)$ one sees that the error is even much smaller, the largest value of $|N /(d q)-S(m, N)|$ being $\approx 29.897$ for $m=1272736$. This is due to the fact that the terms $q$ and $d$ in the $E$-term of (15) come from the Dedekind sums $S(r, q)$ and $S(c, d)$ of $(9)$, which are expected to be much smaller than $d$ and $q$ themselves (cf. (7)). In the following table we list the closest $F$-neighbours $m$ of $N \cdot c / d$ together with $S(m, N)$ and $\Delta=N /(d q)-S(m, N)$. The table shows that the size of the positive spike of the graph $G$ is considerably smaller than that of the negative one here, which is a consequence of $x_{m}=-2 / 11$ for $m=1272729$ but $x_{m}=9 / 11$ for $m=1272730$.

\begin{tabular}{crr|rrc}
\hline$m$ & $S(m, N)$ & \multicolumn{1}{c|}{$\Delta$} & $m$ & $S(m, N)$ & $\Delta$ \\
\hline 1272725 & -3954.081 & 1.506 & 1272730 & 20199.192 & 2.859 \\
1272726 & -5184.021 & -10.792 & 1272731 & 9090.786 & 0.136 \\
1272727 & -7553.322 & -22.447 & 1272732 & 5861.443 & 3.669 \\
1272728 & -13985.594 & -0.441 & 1272733 & 4328.184 & 0.827 \\
1272729 & -90907.864 & -1.364 & 1272734 & 3422.278 & 8.259 \\
\hline
\end{tabular}

REMARK. Although it often happens, it is not always true that $N /(d q)$ is a reasonable approximation of $S(m, N)$ for distant $F$-neighbours, especially if $d$ is small. So take $N=1009, C=1.2$, and $d=1$. Then $m=36$ lies in $I_{0}$; however, $S(m, N) \approx-7.992$, whereas $N /(d q)=N / m \approx 28.028$. If, on the other hand, $C$ has been chosen small enough (say $C=1 / 5$ ), then $N /(d q)$ clearly dominates the error term of (16) for all $F$-neighbours. 


\section{References}

[1] T. M. Apostol, Introduction to Analytic Number Theory, Springer, New York, 1976.

[2] R. Bruggeman, On the distribution of Dedekind sums, in: Contemp. Math. 166, Amer. Math. Soc., 1994, 197-210.

[3] -, Dedekind sums for Hecke groups, Acta Arith. 71 (1995), 11-46.

[4] J. B. Conrey, E. Fransen, R. Klein and C. Scott, Mean values of Dedekind sums, J. Number Theory 56 (1996), 214-226.

[5] K. Girstmair, Dedekind sums with predictable signs, Acta Arith. 83 (1998), 283-294.

[6] L.-K. Hua, Introduction to Number Theory, Springer, Berlin, 1982.

[7] G. Tenenbaum, Introduction to Analytic and Probabilistic Number Theory, Cambridge Univ. Press, Cambridge, 1995.

[8] I. Vardi, Dedekind sums have a limiting distribution, Internat. Math. Res. Notices 1993, no. 1, 1-12.

[9] W. Zhang, A note on the mean square value of the Dedekind sums, Acta Math. Hungar. 86 (2000), 275-289.

Institut für Mathematik

Universität Innsbruck

Technikerstr. 25/7

A-6020 Innsbruck, Austria

E-mail: Kurt.Girstmair@uibk.ac.at

Received on 8.7.2002

and in revised form on 18.9.2002 